\title{
Influência do Tipo de Terapia Antineoplásica sobre Marcadores Antropométricos e Dietéticos em Mulheres Portadoras de Câncer de Mama
}

\author{
Influence of the Type of Cancer Treatment Performed on the Body Composition \\ and Diet of Women with Breast Cancer \\ La Influencia del Tipo de Terapia Antineoplásica sobre Marcadores Antropométricos \\ y de la Dieta de las Mujeres con Cáncer de Mama
}

\author{
Helena Alves de Carvalho Sampaio'; Naiane Matoso de Oliveira² ; Maria Olganê Dantas Sabry³; Antonio Augusto Ferreira Carioca ${ }^{4}$; \\ Luiz Gonzaga Porto Pinheiro ${ }^{5}$
}

\section{Resumo}

Introduçáo: Alteraçôes na situação ponderal, estado nutricional e padrão alimentar são frequentemente citados em pacientes com câncer estando presentes em todas as fases da doença, sendo, entre outras causas, acarretados pelos incômodos e alteraçôes fisiológicas decorrentes do tratamento. Objetivo: Avaliar a influência do tipo de terapia antineoplásica sobre marcadores antropométricos e dietéticos de mulheres portadoras de câncer de mama atendidas em instituiçôes de referência localizadas na cidade de Fortaleza - Ceará. Método: A amostra foi composta de 182 mulheres, distribuídas em três grupos de tratamento, independente da realização prévia de cirurgia: I ( $n=57)$ quimioterapia; II $(\mathrm{n}=59)$ quimioterapia e radioterapia; III $(\mathrm{n}=66)$ sem tratamento. Para análise da situação ponderal, foram aferidos peso, altura e medida de cintura. O peso habitual também foi indagado. Para os dados alimentares, foi utilizado um recordatório de ingestão alimentar de 24 horas. Resultados: Foi detectado um predomínio de excesso ponderal sem diferença estatística entre os grupos. A dieta ingerida pela maioria das entrevistadas foi caracterizada por baixa ingestão energética, de carboidratos e de fibras, participação adequada de fraçóes lipídicas, colesterol e açúcares simples, e excesso de consumo de proteínas, sal e sódio. Tal composição da dieta não foi associada ao tipo de tratamento. Conclusão: No grupo estudado, o tipo de tratamento antineoplásico instituído não teve influência na situação nutricional e alimentar detectada.

Palavras-chave: Neoplasias da Mama/radioterapia; Neoplasias da Mama/quimioterapia; Consumo de Alimentos; Estado Nutricional; Dieta; Antropometria

\footnotetext{
Pesquisa realizada na Maternidade Escola Assis Chateaubriand. Instituto do Câncer do Ceará e Instituto de Prevenção do Câncer do Ceará.

Este estudo foi financiado pela Fundaçáo Cearense de Apoio ao Desenvolvimento Científico e Tecnológico.

${ }^{1}$ Doutora em Farmacologia pela Universidade Federal do Ceará (UFC). Professora Emérita da Universidade Estadual do Ceará (UECE). Mestrado e Doutorado em Saúde Coletiva da UECE. Centro de Ciência da Saúde. Fortaleza (CE), Brasil.

${ }^{2}$ Nutricionista pela UECE. Fortaleza (CE), Brasil.E-mail: naianematoso@uol.com.br.

${ }^{3}$ Doutora em Saúde Pública pela UECE. Professora Adjunta da UECE. Fortaleza (CE), Brasil. E-mail: olgane@oi.com.br.

${ }^{4}$ Nutricionista. Mestrando em Saúde Pública pela Universidade de São Paulo (USP). Fortaleza (CE), Brasil. E-mail: aafc7@hotmail.com.

${ }_{5}^{5}$ Doutor em Cirurgia pela Universidade Federal de Pernambuco (UFPE). Professor Adjunto da UFC. E-mail: geeon.adm@gmail.com.

Enderę̧o para correspondência: Helena Alves de Carvalho Sampaio. Rua Joaquim Nabuco, 500 - apto 402. Fortaleza (CE), Brasil. CEP 60125-120.

E-mail: dr.hard@terra.com.br.
} 


\section{INTRODUÇÃO}

O câncer de mama foi recentemente apontado como a primeira causa de mortalidade mundial, sendo essa doença considerada um problema de saúde pública ${ }^{1-2}$. É o tipo de câncer mais prevalente em mulheres das regióes Sudeste (69/100 mil), Sul (65/100 mil), Centro-Oeste (48/100 mil) e Nordeste (32/100 mil), sem considerar os tumores da pele não melanoma. Para 2012, estima-se, para o Brasil, 52.680 novos casos desse câncer, sendo também o mais comum no mundo, tanto em países em desenvolvimento quanto em países desenvolvidos ${ }^{3}$.

Atualmente, são disponíveis vários tipos de tratamento contra a doença, sejam para aplicação de forma isolada, sejam em associação, como cirurgia, radioterapia, hormonioterapia e quimioterapia ${ }^{4}$. A terapia considerada adjuvante tem o objetivo de diminuir a chance de recidiva local e sistêmica, destacando-se aí a cirurgia e a hormonioterapia; a radioterapia diminui a chance de recidiva local e a quimioterapia a recidiva sistêmicas.

A cirurgia é subdividida em conservadora e não conservadora, sendo a cirurgia conservadora indicada para tumores com extensóes menores, e a não conservadora indicada quando não é possível assegurar a obtenção de margens livres, em função da extensão ou multicentricidade do tumor ${ }^{4}$.

A hormonioterapia ou terapia endócrina adjuvante é um método que consiste no uso de substâncias semelhantes ou inibidoras de hormônios para tratar as neoplasias que são dependentes desses hormônios ${ }^{6}$.

A radioterapia é um método que utiliza radiaçóes para destruir um tumor ou impedir o aumento das células tumorais. Antes da cirurgia, é utilizada para reduzir o tamanho do tumor e, após, para destruir as células remanescentes. Pode ser utilizada de forma isolada ou associada à quimioterapia ou outros tipos de tratamentos. Já quimioterapia é um tratamento para neoplasias que utiliza compostos químicos. Pode ser aplicada a outras doenças; mas, quando utilizada no tratamento de câncer, é chamada de quimioterapia antineoplásica ou quimioterapia antiblástica ${ }^{4}$.

Alteraçôes na situaçáo ponderal, estado nutricional e padrão alimentar são frequentemente citados em pacientes com câncer, estando presentes em todas as fases da doença, sendo, dentre outras causas, acarretados pelos incômodos e alteraçóes fisiológicas decorrentes do tratamento ${ }^{7}$, que serão citadas mais amplamente no decorrer desse estudo.

Considerando a escassez de estudos avaliando a repercussão dos diferentes tratamentos sobre a composição corporal e comportamento alimentar e, consequentemente, sobre o risco de recidiva da doença, este artigo tem como objetivo avaliar a influência do tipo de terapia antineoplásica sobre marcadores antropométricos e dietéticos de mulheres portadoras de câncer de mama atendidas em instituiçóes de referência localizadas na cidade de Fortaleza, Ceará.

\section{MÉTODO}

Trata-se de um estudo do tipo descritivo-exploratório, com abordagem quantitativa e comparativa, tendo como foco central a avaliação das repercussôes nutricionais em mulheres portadoras de câncer de mama. O mesmo foi submetido e aprovado pelo Comitê de Ética em Pesquisa da Instituição responsável pela execução do estudo (CAAE - 0025.0.038.036-08).

A amostra foi composta de 182 mulheres, representada aleatoriamente por mulheres que buscaram atendimento preventivo ou terapêutico nas três instituiçóes de referência na prevenção e tratamento do câncer de mama na cidade de Fortaleza, Ceará, nos anos de 2007 a 2009, sendo todas as pacientes atendidas pelo Sistema Único de Saúde (SUS), independente da instituição. Foram excluídas aquelas que possuiam alguma outra neoplasia associada ou alguma doença crônica grave. Todas assinaram um termo de consentimento livre e esclarecido antes da entrevista.

Essas mulheres foram avaliadas até seis meses após o tratamento, sendo distribuídas em três grupos de acordo com a modalidade terapêutica instituída: Grupo I - as que tinham recebido apenas quimioterapia (além da realização ou não de cirurgia); Grupo II - aquelas que haviam recebido apenas quimioterapia e radioterapia (além da realização ou não de cirurgia); Grupo III - aquelas sem tratamento (exceto cirurgia).

Sendo assim, inicialmente foi realizada a caracterizaçáo do grupo, considerando o tipo de tratamento realizado. A amostra total de 182 mulheres foi subdividida em $31,3 \%$ do grupo I ( $n=57) ; 32,4 \%$ do grupo II ( $n=59)$; e $36,3 \%$ do grupo III ( $n=66)$.

Foi determinado o estado nutricional de cada entrevistada e utilizado como parâmetro o índice de massa corpórea (IMC), definido pela relação peso/altura ${ }^{2}\left(\mathrm{Kg} / \mathrm{m}^{2}\right)$, sendo adotada a classificação da World Health Organization $(\mathrm{WHO})^{8}$. Para este estudo, a categoria obesidade não foi dividida segundo os graus de excesso, sendo então estabelecida a seguinte classificação: baixo peso $\left(<18,5 \mathrm{Kg} / \mathrm{m}^{2}\right)$, eutrofia $\left(18,5-4,9 \mathrm{Kg} / \mathrm{m}^{2}\right)$, pré-obesidade ou sobrepeso $(25,0-29,9$ $\left.\mathrm{Kg} / \mathrm{m}^{2}\right)$ e obesidade $\left(\geq 30,0 \mathrm{Kg} / \mathrm{m}^{2}\right)$. O peso e a altura foram obtidos com auxílio de uma balança antropométrica mecânica, da própria instituição, marca Filizola ${ }^{\circledR}$, com capacidade de $150 \mathrm{Kg}$ e sensibilidade de $100 \mathrm{~g}$ e de 2,0 m, com sensibilidade de $0,5 \mathrm{~cm}$. O peso habitual (antes do tratamento) também foi indagado e confrontado com o peso atual para verificar perda ou ganho de peso. 
Foi ainda avaliada a medida da circunferência da cintura (CC), obtida através de fita métrica inextensível de $150 \mathrm{~cm}$ de comprimento, com sensibilidade de $0,5 \mathrm{~cm}$. Adotou-se como protocolo e parâmetro de normalidade a proposição da $\mathrm{WHO}^{8}$.

A análise de consumo alimentar foi obtida a partir de um recordatório alimentar de 24 horas, no qual foi relatada a ingestáo referente ao dia anterior. Aliou-se a esse um questionário de frequência alimentar, a fim de verificar a variedade e a necessidade ou não de realização de um segundo recordatório alimentar. Os dados relativos ao consumo alimentar foram fornecidos em medidas caseiras. Após essa transformação, os dados foram inseridos no software DietWin ${ }^{\bullet}$ Profissional 2.0 para determinar a composiçáo da dieta dessas mulheres. Foram analisados: energia, proteínas, carboidratos (discriminando ingestáo de açúcares simples e fibra alimentar), lipídios (discriminando totais, gordura saturada e colesterol), sódio e quantidade habitual de sal, determinando-se as médias de cada grupo.

O parâmetro adotado para estabelecer se a ingestáo energética estava adequada foi a comparação da encontrada com as recomendaçôes energéticas previstas pela Dietary Reference Intake (DRI) ${ }^{9}$. Os macros, micronutrientes, e consumo energético foram avaliados segundo parâmetros para uma dieta saudável ${ }^{10}$, sendo recomendados $2.000 \mathrm{kcal}$ de consumo energético, proteína de 10 a $15 \%$, carboidrato de 55 a $75 \%$, lipídio de 15 a 30\% e gordura saturada e açúcar simples de $<10 \%$ do valor energético total; colesterol até $300 \mathrm{~g} / \mathrm{dia}$, fibra no mínimo $25 \mathrm{~g} /$ dia e sal até $5 \mathrm{~g} /$ dia.

Os dados foram analisados utilizando-se o Excel e o programa estatístico Predictive Analytics Software for Windows (PASW), versão 17.0, sendo adotado $\mathrm{p}<0,05$, como nível de significância. Os dados gerais foram analisados de forma descritiva usando-se as frequências (absoluta e percentual) e as medidas paramétricas (média e desvio-padrão (DP)).

Foi utilizado o teste Anova para comparar as médias antropométricas e dietéticas dos grupos de tratamento, observando-se anteriormente o teste de normalidade de Kolmogorov-Sminorv.

\section{RESULTADOS}

A idade média global do grupo foi de 51,8 anos, (30-78 anos), sendo a do grupo I de 50,2 anos (30-77 anos); do grupo II de 51,3 anos (35-71 anos); e do grupo III de 53,6 anos (33-78 anos).

A Tabela 1 mostra os dados relativos ao estado nutricional dos grupos segundo o IMC. Observa-se que nos três grupos há uma alta prevalência de excesso ponderal, sendo um percentual levemente maior no grupo III $(72,7 \%)$, seguido do grupo II $(71,2 \%)$ e por último grupo I $(68,4 \%)$.

$\mathrm{Na}$ tabela 2, são apresentadas as médias dos indicadores antropométricos obtidas segundo os grupos estudados. Não foi observada diferença estatística entre os grupos.

Tabela 1. Distribuição das mulheres estudadas segundo o estado nutricional determinado pelo IMC e grupo de tratamento. Fortaleza, 2007-2009

\begin{tabular}{|c|c|c|c|c|c|c|c|c|}
\hline \multirow{3}{*}{$\begin{array}{c}\text { Classificação do } \\
\text { IMC* }\end{array}$} & \multicolumn{8}{|c|}{ Grupo de tratamento** } \\
\hline & \multicolumn{2}{|c|}{$\mathbf{I}$} & \multicolumn{2}{|c|}{ II } & \multicolumn{2}{|c|}{ III } & \multicolumn{2}{|c|}{ Total } \\
\hline & $\mathbf{N}$ & $\%$ & $\mathbf{N}$ & $\%$ & $\mathbf{N}$ & $\%$ & $\mathbf{N}$ & $\%$ \\
\hline Baixo Peso & - & - & 2 & 3,4 & - & - & 2 & 1,1 \\
\hline Eutrófico & 18 & 31,6 & 15 & 25,4 & 18 & 27,3 & 51 & 28,0 \\
\hline Sobrepeso & 28 & 49,1 & 25 & 42,4 & 27 & 40,9 & 80 & 44,0 \\
\hline Obesidade & 11 & 19,3 & 17 & 28,8 & 21 & 31,8 & 49 & 26,9 \\
\hline Total & 57 & 100,0 & 59 & 100,0 & 66 & 100,0 & 182 & 100,0 \\
\hline
\end{tabular}

Legendas: *Categorizado segundo IMC; ${ }^{* *}$ Grupo I (quimioterapia), grupo II (quimioterapia + radioterapia), e grupo III (sem tratamento, exceto cirurgia).

Tabela 2. Perfil antropométrico das mulheres estudadas segundo grupo de tratamento. Fortaleza, 2007-2009

\begin{tabular}{|c|c|c|c|c|c|}
\hline \multirow{3}{*}{ Antropometria } & \multicolumn{5}{|c|}{ Grupo de tratamento* } \\
\hline & $\mathbf{I}$ & II & III & $\mathbf{p}^{* *}$ & Total \\
\hline & Média (DP) & Média (DP) & Média (DP) & & Média (DP) \\
\hline Peso (kg) & $63,5(10,9)$ & $63,5(12,0)$ & $66,4(11,2)$ & 0,246 & $64,5(11,4)$ \\
\hline $\mathrm{CC}(\mathrm{cm})$ & $89,6(10,2)$ & $90,0(10,3)$ & $92,2(11,4)$ & 0,343 & $90,7(10,7)$ \\
\hline $\mathrm{IMC}\left(\mathrm{kg} / \mathrm{m}^{2}\right)$ & $26,8(4,2)$ & $27,3(4,8)$ & $28,5(4,7)$ & 0,089 & $27,6(4,6)$ \\
\hline
\end{tabular}

Legendas: *Grupo I (quimioterapia), grupo II (quimioterapia + radioterapia), e grupo III (sem tratamento, exceto cirurgia); **Teste Anova. 
A relação entre o peso atual e o habitual foi investigada com o objetivo de verificar se houve perda, ganho ou manutenção do peso (Tabela 3). Ressalta-se que algumas participantes não souberam referir o peso habitual, sendo 5 do grupo I, 4 do grupo II e 16 do grupo III.

$\mathrm{Na}$ comparaçáo do peso atual com o peso habitual autorrreferido, constatou-se pequeno ganho de peso no grupo todo, sendo que o grupo I exibiu uma média de $+0,06 \mathrm{Kg}$; o grupo II exibiu uma média de $+1,33 \mathrm{Kg}$; e o grupo III uma média de $+1,34 \mathrm{Kg}$. Não houve diferença estatística entre os grupos.

A composição da dieta do grupo está descrita em médias e desvios-padrão na Tabela 4. A composição nutricional das dietas foi semelhante, observando-se diferença estatística apenas no consumo de gordura saturada entre os grupos II e III ( $\mathrm{p}=0,023)$, embora a ingestão de gordura saturada pelos três grupos foi dentro dos parâmetros de normalidade. As dietas mostraram-se inadequadas no que tange às fibras e carboidratos, ingeridos em quantidade insuficiente; e quanto ao sal e sódio, ingeridos em excesso.

\section{DISCUSSÃO}

Os resultados mostram que, em todos os grupos, houve predomínio de excesso ponderal, o que pode acarretar outras complicaçóes como doenças cardiovasculares e dislipidemia, além de representar risco para recidiva de câncer de mama e para o surgimento de outras neoplasias ${ }^{11}$. Assim, é importante a intervenção educativa nos três grupos.

Tal excesso ponderal acarreta um aumento no nível de hormônio circulante e fatores pró-inflamatórios, como: fator de necrose tumoral, interleucinas e proteína $\mathrm{C}$ reativa, esses induzem à progressáo do ciclo celular e inibem a apoptose, aumentando o risco de desenvolver o câncer ${ }^{12}$.

Em estudo recente, Nechuta et al. ${ }^{13}$, em uma pesquisa que foi constituída por quatro estudos de coorte com mulheres norte-americanas e chinesas $(n=18.314)$ sobreviventes de câncer de mama, entre 20 a 83 anos, que tiveram câncer nos anos de 1976 a 2006, constataram, nos dois anos pós-diagnóstico, que 50,5\% apresentavam

Tabela 3. Distribuição das mulheres estudadas segundo evolução ponderal em relação ao peso habitual e grupo de tratamento. Fortaleza, 2007-2009

\begin{tabular}{|c|c|c|c|c|c|c|c|c|}
\hline \multirow{3}{*}{ Evolução ponderal* } & \multicolumn{8}{|c|}{ Grupo de tratamento** } \\
\hline & \multicolumn{2}{|c|}{$\mathbf{I}$} & \multicolumn{2}{|c|}{ II } & \multicolumn{2}{|c|}{ III } & \multicolumn{2}{|c|}{ Total } \\
\hline & $\mathbf{N}$ & $\%$ & $\mathbf{N}$ & $\%$ & $\mathbf{N}$ & $\%$ & $\mathbf{N}$ & $\%$ \\
\hline Perda & 27 & 51,9 & 20 & 36,4 & 22 & 44,0 & 69 & 44,0 \\
\hline Ganho & 22 & 42,3 & 34 & 61,8 & 26 & 52,0 & 82 & 52,2 \\
\hline Manutenção & 3 & 5,8 & 1 & 1,8 & 2 & 4,0 & 6 & 3,8 \\
\hline Total & 52 & 100,0 & 55 & 100,0 & 50 & 100,0 & 157 & 100,0 \\
\hline
\end{tabular}

Legendas: *Em relação ao peso habitual; ${ }^{* *}$ Grupo I (quimioterapia), grupo II (quimioterapia + radioterapia), e grupo III (sem tratamento, exceto cirurgia).

Tabela 4. Composição da dieta das mulheres estudadas segundo grupo de tratamento. Fortaleza, 2007-2009

\begin{tabular}{l|c|c|c|c|c}
\hline \multirow{2}{*}{ Consumo Alimentar } & \multicolumn{5}{|c}{ Grupo de tratamento* } \\
\cline { 2 - 6 } & I & II & III & p** & Total \\
\cline { 2 - 6 } & Média (DP) & Média (DP) & Média (DP) & Média (DP) \\
\hline Energia (kcal) & $1562,1(590,9)$ & $1587,3(626,3)$ & $1460,0(546,0)$ & 0,440 & $1522,5(586,5)$ \\
\hline Carboidrato (\%) & $52,7(10,0)$ & $50,3(10,8)$ & $52,2(9,9)$ & 0,398 & $51,7(10,2)$ \\
\hline Açúcar simples (\%) & $10,7(9,0)$ & $9,8(10,6)$ & $9,0(7,5)$ & 0,584 & $9,8(9,0)$ \\
\hline Proteína (\%) & $18,0(6,7)$ & $18,4(6,4)$ & $19,3(7,8)$ & 0,593 & $18,6(7,0)$ \\
\hline Lipídio (\%) & $29,3(7,1)$ & $31,4(8,8)$ & $28,1(9,3)$ & 0,094 & $29,5(8,5)$ \\
\hline Gordura saturada (\%) & $8,2(3,2)$ & $9,23(5,0)$ & $7,5(3,0)$ & 0,030 & $8,3(3,8)$ \\
\hline Colesterol (mg) & $208,3(158,4)$ & $214,5(159,3)$ & $200,9(145,9)$ & 0,885 & $207,6(153,5)$ \\
\hline Fibra (g) & $17,3(9,6)$ & $16,1(8,3)$ & $17,4(10,1)$ & 0,713 & $16,9(9,3)$ \\
\hline Sódio (g) & $4,2(2,4)$ & $4,3(2,8)$ & $5,0(3,9)$ & 0,286 & $4,5(3,1)$ \\
\hline Sal dietético (g) & $8,6(6,1)$ & $8,8(7,0)$ & $10,6(10,0)$ & 0,307 & $9,3(7,7)$ \\
\hline
\end{tabular}

Legendas: *Grupo I (quimioterapia), grupo II (quimioterapia + radioterapia), e grupo III (sem tratamento, exceto cirurgia); ${ }^{* *}$ Teste Anova. 
excesso ponderal, sendo que 17,9\% eram obesas. Esse achado torna-se preocupante, pois, assim como o presente estudo apresentou elevada prevalência de excesso ponderal, pode influenciar no desenvolvimento/recidiva do câncer.

Em um estudo prospectivo com 99.039 mulheres na pós-menopausa, entre 50 a 71 anos, em seis Estados dos EUA, houve 2.111 casos incidentes de câncer de mama, sendo observado que o IMC atual estava fortemente associado com a doença avançada (metástases) em relação à não avançada $(\mathrm{p}=0,009)$. Também avaliaram que a $\mathrm{CC} \geq 97 \mathrm{~cm}$ elevou o risco da doença e que uma $\mathrm{CC} \geq 103 \mathrm{~cm}$ levou a um aumento de 55\% no risco de desenvolver o câncer de mama ${ }^{14}$.

Considerando os presentes resultados relativos à evolução ponderal, percebe-se que uma minoria manteve seu peso habitual. As prevalências tanto de ganho ponderal como de perda de peso foram altas, embora a média de cada grupo tenha resultado em aquisição de peso. A literatura aponta motivos tanto para ganho como para perda ponderal, como será discutido.

Beasley et al. ${ }^{15}$, avaliando 13.302 pacientes sobreviventes de câncer de mama, em média 23 meses após o diagnóstico, nos EUA e na China, encontraram que mulheres com $10 \%$ ou mais de aumento de peso antes do diagnóstico tiveram 14\% mais chance de recidiva da doença. Segundo os autores, as mulheres mais magras tiveram um maior ganho de peso. Outro aspecto, esse não avaliado no presente estudo, mas importante para planejamento de atividades educativas para tais mulheres sobreviventes, foi de que pacientes com pelo menos 2,5 horas de atividade física por semana tiveram redução de $27 \%$ em todas as causas de mortalidade e redução de $25 \%$ de mortalidade por câncer de mama.

No grupo I, houve alta prevalência de perda ponderal, o que pode ser resultante dos efeitos colaterais sobre o aparelho digestivo decorrentes dos esquemas de drogas quimioterápicas utilizados, bem como náuseas e vômitos pós-quimioterapia, que podem causar fissuras esofágicas, anorexia com má-nutrição, distúrbios hidroeletrolíticos e até mesmo a recusa dos pacientes em prosseguirem nos ciclos quimioterápicos ${ }^{16}$. Ishikawa et al. ${ }^{17}$, avaliando a presença de fadiga em três grupos de mulheres com câncer de mama (após tratamento cirúrgico, em quimioterapia e/ou radioterapia e em pacientes que não realizaram nenhum tratamento adjuvante), detectaram aumento da prevalência e gravidade da fadiga durante os ciclos de quimioterapia, sendo mais evidente nos primeiros dias após quimioterapia em relação ao período entre os ciclos.

Por outro lado, o medo da desnutrição e de possíveis alteraçôes gastrointestinais e alimentares associadas ao tratamento pode levar o paciente a mudar a sua rotina de alimentação, sendo, em grande parte deles, uma mudança inadequada que pode, paradoxalmente, acarretar um ganho ponderal, pois a pessoa se força a $\operatorname{comer}^{18}$. Tal ocorrência pode explicar o ganho ponderal observado em parte do grupo I.

Em relação ao grupo II, poder-se-ia esperar maior prevalência de perda ponderal, pois se associaram dois tratamentos agressivos: quimioterapia e radioterapia. No entanto, apesar da falta de comprovação estatística, o percentual de mulheres com ganho ponderal pareceu ser maior, indicando que poderia ser apropriado realizar a investigação com maior número de pacientes, a fim de se descartar com certeza uma maior presença de ganho ponderal nesse grupo.

No caso do grupo III, alteraçôes psicológicas podem ser uma hipótese para explicar a perda ou o ganho de peso; pois, segundo Hoffmann et al. ${ }^{19}$, esse tipo de paciente cirúrgico passa por ansiedade e depressão decorrente da perda de um órgão ou parte do corpo, o que pode tanto resultar em comportamento dirigido para o excesso alimentar como acarretar anorexia.

Como a cirurgia é um procedimento comumente adotado, ainda que se associe à quimioterapia e/ou à radioterapia, a alteração de comportamento alimentar poderia ocorrer nos três grupos, tanto se traduzindo em excesso, como em deficiente ingestão, dependendo de como a paciente lida com a doença e seu tratamento. Isso explica a distribuição dos três grupos em ganhar ou perder peso.

Por outro lado, a ingestáo calórica foi predominantemente insuficiente em ambos os grupos, o que fica em desacordo com a alta prevalência de excesso ponderal. Duas hipóteses podem ser elaboradas: relativa à metodologia utilizada $\mathrm{e}$ às interaçôes decorrentes do tratamento. A metodologia de inquérito alimentar utilizada para obtenção de dados, apesar de ser recomendada, é associada a falhas, dependendo da habilidade tanto do entrevistado como do entrevistador em estimar as porçôes reais dos alimentos, podendo estar associada a informaçóes não fidedignas ${ }^{20}$. Sabe-se, ainda, que a subestimação de consumo é frequente entre portadores de excesso ponderal ${ }^{20}$. Quanto às interaçóes decorrentes do tratamento, pode ser que as mulheres estivessem vivenciando anorexia na época da coleta de dados, mas a redução da ingestáo ainda náo houvesse levado à perda ponderal. No entanto, como a literatura mostra que costuma ocorrer ganho de peso nesse tipo de paciente ${ }^{21}$, é mais provável que tenha ocorrido subestimaçáo de consumo no momento da coleta da informação.

As mulheres ingeriram uma dieta hiperproteica e hipoglicídica, baixa em fibras e com alto teor de sódio. Comparando-se com dados populacionais recentes, detectados pela Pesquisa de Orçamentos Familiares 2008- 
2009 (POF) ${ }^{22}$, mulheres entre 19 a 59 anos apresentaram ingestão similar às mulheres do presente estudo, à exceção da ingestáo mais elevada de açúcares e menos excessiva de sódio, com média de consumo energético de $1.683 \mathrm{kcal}, 16,9 \%$ de proteína, 56,7\% de carboidrato, 26,3\% de lipídios, 9,1\% de gordura saturada, $235,1 \mathrm{mg}$ de colesterol, 17,3 g de fibra alimentar, $19,9 \%$ de açúcar simples e $2.782,4$ mg de sódio. Ainda segundo a POF, na faixa etária de 60 ou mais anos, as médias de consumo foram similares às mulheres mais jovens, portanto com as mesmas diferenças em relação ao grupo aqui avaliado: $1.448 \mathrm{kcal}$, com $17,5 \%$ de proteína; $57,6 \%$ de carboidrato; $25,2 \%$ de lipídios; $9,0 \%$ de gordura saturada; $189,1 \mathrm{mg}$ de colesterol; $16,6 \mathrm{~g}$ de fibra alimentar; $19,4 \%$ de açúcar simples; e $2.481,3 \mathrm{mg}$ de sódio.

Outro ponto de discussão para avaliação do consumo energético e proteico poderia ser a comparaçáo com as diretrizes do Consenso Nacional de Nutrição Oncológica ${ }^{23}$. Segundo esse Consenso, como a populaçáo do presente estudo constitui-se, principalmente, de mulheres com excesso ponderal, seriam recomendados, para quimioterapia e radioterapia, 21-25 Kcal/Kg/dia para obesas e 25-30 $\mathrm{Kcal} / \mathrm{Kg} /$ dia para manutenção do peso e recomendação proteica de 1,0-1,2 gramas $/ \mathrm{Kg} /$ dia para tratamento sem complicações. Essas recomendações não foram utilizadas, pois nos serviços onde os dados foram coletados essa conduta não integra a rotina de atendimento durante $\mathrm{e}$ pós-tratamento, sendo utilizada a recomendaçáo genérica de uma dieta saudável, razão pela qual aqui foram adotadas as recomendaçôes do Guia Alimentar para a População Brasileira $^{10}$. Mesmo assim, percebe-se, pelos dados encontrados, que as mulheres estavam atendendo à ingestáo calórica e proteica constante no citado Consenso ${ }^{23}$.

Um estudo prospectivo publicado recentemente, também com americanas, entre 20 e 79 anos, sobreviventes do câncer de mama $(n=4.441)$, encontrou que as mulheres, cujo consumo médio de calorias provenientes de gordura saturada era de $13 \%$, tiveram um risco $41 \%$ maior de morte por qualquer causa em comparação as que apresentaram consumo médio de $7 \%(\mathrm{p}=0,03)$. Tal pesquisa também verificou que o quintil superior da ingestão de gordura trans levou a um aumento de $78 \%$ no risco de morte em comparaçáa com o menor quintil $(\mathrm{p}=0,01)$. Esse achado demonstra que a qualidade da gordura consumida foi mais importante do que o próprio consumo de gordura, pois a ingestão de gordura total não foi associada com a sobrevida ${ }^{24}$.

Os dados apontam para uma náo influência do tratamento na busca por alimentos específicos, contrariando relatos da literatura ${ }^{21}$. Malzyner e Caponero ${ }^{18}$ referem que o tratamento quimioterápico pode levar a gosto metálico e alteraçóes na percepção do gosto dos alimentos, com diminuição da percepção do sal e do sabor amargo nos alimentos, e uma preferência por alimentos doces. Avaliando-se, sob essa ótica, se houve alguma influência, pode ter sido devida à quimioterapia nos grupos I e II, no que tange ao uso excessivo de sal e sódio; pois, com a redução da percepção, tende-se a aumentar a quantidade para conferir melhor sabor aos alimentos. Mas essa explicaçáo não se aplica ao grupo III, que também teve consumo elevado desses nutrientes, inclusive aparentemente maior nesse grupo, embora sem significado estatístico, o que também aponta para a necessidade de estudos com amostra maior. Outro aspecto que merece investigação futura é o relativo à ingestão de açúcares, que, embora adequada, se mostrou bem inferior à média nacional detectada na POF. Se houver interferência, seria reduzindo a aceitação de alimentos doces.

Velentzis et al. ${ }^{25}$, avaliando 1.560 sobreviventes do câncer de mama do Reino Unido antes e após diagnóstico, verificaram um aumento significante de frutas, legumes, cereais integrais, fibra alimentar e carnes magras $(\mathrm{p}<0,05)$ e, por outro lado, diminuição de energia consumida, gordura total, saturada, monoinsaturada, poli-insaturada, proteína, carboidrato e sódio. No momento após o diagnóstico, houve aumento também da utilização de óleo de peixe e suplemento de vitaminas e minerais. Assim, o diagnóstico de câncer de mama pode modificar hábitos e estilo de vida. Tais modificaçóes podem partir por iniciativa da própria sobrevivente, ser fruto de orientaçóes efetuadas pelo serviço de saúde ou ambas.

\section{CONCLUSÃO}

Todos os grupos avaliados apresentaram uma alta prevalência de excesso ponderal, com acúmulo de gordura abdominal, este estimado pela medida da CC. Tal situação não foi associada ao tipo de tratamento antineoplásico instituído. Com relação à evolução ponderal, foi detectado tanto ganho como perda ponderal nos três grupos, também não sendo possível estabelecer relação com o tipo de tratamento realizado.

O consumo alimentar foi predominantemente caracterizado por uma ingestão energética insuficiente, deficiente contribuiçáo de carboidratos e fibras, adequada ingestão de fraçôes lipídicas, colesterol e açúcares simples, e excesso de consumo de proteínas, sal e sódio. Tal composição da dieta não foi associada à da terapêutica instituída.

No grupo estudado o tipo de terapia antineoplásica utilizado não exerceu influência em marcadores antropométricos e dietéticos. As inadequaçóes encontradas demandam açóes educativas dirigidas a todas as sobreviventes de câncer de mama, independente do tipo de tratamento utilizado. 


\section{CONTRIBUIÇÕES}

Helena Alves de Carvalho Sampaio participou da concepçáo e planejamento do projeto, redaçáo final do manuscrito; Naiane Matoso de Oliveira participou da obtenção dos dados e redação final do manuscrito; Maria Olganê Dantas Sabry e Luiz Gonzaga Porto Pinheiro participaram da redaçáo final do manuscrito; Antonio Augusto Ferreira Carioca participou da análise e redação final do manuscrito.

\section{Declaraçáo de Conflito de Interesses: Nada a Declarar. \\ REFERÊNCIAS}

1. Garófolo A, Avesani CM, Camargo KG, Barros ME, Silva SRJ, Taddei JAAC, et al. Dieta e câncer: um enfoque epidemiológico. Rev nutr. 2004;17(4):491-505.

2. Guerra MR, Moura Gallo CV, Mendonça GAS. Risco de câncer no Brasil: tendências e estudos epidemiológicos mais recentes. Rev bras cancerol. 2005;51(3):227-34.

3. Instituto Nacional de Câncer José Alencar Gomes da Silva. Estimativa 2012: incidência de câncer no Brasil. Rio de Janeiro: INCA; 2011. 118 p.

4. Instituto Nacional de Câncer (Brasil). Controle do câncer de mama: documento de consenso. Rio de Janeiro: INCA; 2004. 36 p.

5. Del Giglio A, Iyeyasu H. Câncer de mama. In: Lopes A, Iyeyasu H, Lopes LF, Almeida ES, Castro RMRPS, [organizadores]. Oncologia para a graduação. Ribeirão Preto: Tecmedd; 2005. p. 285-94.

6. Brito C, Portela MC, Vasconcellos MTL. Assistência oncológica pelo SUS a mulheres com câncer de mama no Estado do Rio de Janeiro. Rev Saúde Públ. 2005;39(6):874-81.

7. Berteretche MV, Dalix AM, d'Ornano AM, Bellisle F, Khayat D, Faurion A. Decreased taste sensitivity in cancer patients under chemotherapy. Support Care Cancer. 2004;12(8):571-6.

8. World Health Organization. Obesity: preventing and managing the global epidemic: report of a WHO Consultation on Obesity, Geneva, 3-5 June 1997. Geneva: World Health Organization; 1998. (Technical report series; n. 894).

9. Otten JJ, Hellwig JP, Meyers LD, editors. DRI, dietary reference intakes: the essential guide to nutrient requirements. Washington: National Academies Press; c2006. 543 p.

10. Brasil. Ministério da Saúde. Secretaria de Atenção à Saúde. Coordenação Geral da Política de Alimentação e Nutrição. Guia alimentar para a população brasileira: promovendo a alimentação saudável. Brasília: Ministério da Saúde; 2006. 210 p. (Série A. Normas e manuais técnicos).
11. Martins IS, Marinho SP. O potencial diagnóstico dos indicadores da obesidade centralizada. Rev Saúde Públ. 2003;37(6):760-7.

12. World Cancer Research Fund; American Institute for Cancer Research. Food, nutrition, physical activity, and the prevention of cancer: a global perspective. Washington: American Institute for Cancer Research; 2007. 517 p. A project of World Cancer Research Fund International.

13. Nechuta SJ, Caan BJ, Chen WY, Flatt SW, Lu W, Patterson RE, et al. The After Breast Cancer Pooling Project: rationale, methodology, and breast cancer survivor characteristics. Cancer Causes Control. 2011;22(9):1319-31.

14. Ahn J, Schatzkin A, Lacey JV Jr, Albanes D, BallardBarbash R, Adams KF, et al. Adiposity, adult weight change, and postmenopausal breast cancer risk. Arch Intern Med. 2007;167(19):2091-102.

15. Beasley JM, Kwan ML, Chen WY, Weltzien EK, Kroenke $\mathrm{CH}, \mathrm{Lu} \mathrm{W}$, et al. Meeting the physical activity guidelines and survival after breast cancer: findings from the after breast cancer pooling project. Breast Cancer Res Treat. 2012;131(2):637-43.

16. Silva MPN. Síndrome da anorexia-caquexia em portadores de câncer. Rev bras cancerol. 2006;52(1):59-77.

17. Ishikawa NM, Derchain SFM, Thuler LCS. Fadiga em pacientes com câncer de mama em tratamento adjuvante. Rev bras cancerol. 2005;51(4):313-8.

18. Malzyner A, Caponero R. Consequências nutricionais do tratamento quimioterápico. In: Waitzberg DL, editor. Dieta, nutrição e câncer. São Paulo: Atheneu; 2004. p. 399-406.

19. Hoffmann FS, Müller MC, Rubin R. A mulher com câncer de mama: apoio social e espirituralidade. Mudanças - Psicologia da Saúde. 2006;14(2):143-50.

20. Fisberg RM, Slater Villar B, Marchioni DML, Martini LA. Inquéritos alimentares: métodos e bases científicos. Barueri: Manole; 2005. 334 p.

21. Harvie MN, Campbell IT, Baildam A, Howell A. Energy balance in early breast cancer patients receiving adjuvant chemotherapy. Breast Cancer Res Treat. 2004;83(3):201-10.

22. Instituto Brasileiro de Geografia e Estatística. Pesquisa de orçamentosfamiliares 2008-2009: análise do consumo alimentar pessoal no Brasil. Rio de Janeiro: IBGE; 2011. 138 p.

23. Instituto Nacional de Câncer (Brasil). Consenso nacional de nutrição oncológica. Rio de Janeiro: INCA; c2009. 125 p.

24. Beasley JM, Newcomb PA, Trentham-Dietz A, Hampton JM, Bersch AJ, Passarelli MN, et al. Post-diagnosis dietary factors and survival after invasive breast cancer. Breast Cancer Res Treat. 2011;128(1):229-36.

25. Velentzis LS, Keshtgar MR, Woodside JV, Leathem AJ, Titcomb A, Perkins KA, et al. Significant changes in dietary intake and supplement use after breast cancer diagnosis in a UK multicentre study. Breast Cancer Res Treat. 2011;128(2):473-82. 


\section{Abstract}

Introduction: Changes in weight status, nutritional status and dietary patterns are often cited in patients with cancer, being present at all stages of the disease, among other causes brought about by uncomfortable and physiological changes resulting from the treatment. Objective: To evaluate the influence of the anti-neoplastic therapy on anthropometric and dietary markers of women with breast cancer treated at referral institutions in the city of Fortaleza - Ceará. Method: The sample consisted of 182 women, divided into three treatment groups, regardless of any previous surgery: I $(n=57)$ chemotherapy; II ( $n=59)$ chemotherapy and radiation therapy, III $(n=66)$ without treatment. For analysis of the weight status, we measured weight, height and waist circumference. The usual weight was also questioned. For food information a 24-hour intake recall was used. Results: A prevalence of excess weight with no statistical difference between groups was detected. The diet consumed by most of the interviewees was characterized by low energy intake of carbohydrates and fiber, appropriate intake of lipids, cholesterol and simple sugars, and excess consumption of protein, salt and sodium. Conclusion: Such composition of the diet was not associated with the type of treatment. In the group studied, the type of antineoplastic treatment had no influence on the food and nutrition status detected. Key words: Breast Neoplasms/radiotherapy; Breast Neoplasms/drug therapy; Food Consumption; Nutritional Status; Diet; Anthropometry

\section{Resumen}

Introducción: Los cambios en la situación ponderal, estado nutricional y los hábitos alimentarios son citados a menudo en pacientes con cáncer y está presente en todas las etapas de la enfermedad, entre otras razones provocadas por cambios incómodos y fisiológicos resultantes del tratamiento. Objetivo: Evaluar la influencia de la terapia antineoplásica de los indicadores antropométricos y dietéticos de las mujeres con cáncer de mama tratadas en un centro de referencia ubicadas en la ciudad de Fortaleza - Ceará. Método: La muestra consistió en 182 mujeres, divididas en tres grupos de tratamiento, independientemente de cualquier cirugía anterior: I ( $\mathrm{n}=57)$ la quimioterapia; II ( $\mathrm{n}=59)$ la quimioterapia y la radioterapia, III $(\mathrm{n}=66)$ sin el tratamiento. Para el análisis de la situación ponderal se midió peso, talla y circunferencia de la cintura. El peso normal también fue averiguado. Para los datos alimentares, fue utilizado un recordatorio de ingestión alimentaria de 24 horas. Resultados: Se ha detectado una prevalencia de exceso de peso, sin diferencias estadísticas entre los grupos. La dieta consumida por la mayoría de los entrevistados se ha caracterizado por la baja ingestión energética, de carbohidratos y de fibras, participación adecuada de fracciones de lipídeos, colesterol y azúcares simples, y el consumo excesivo de proteínas, sal y sodio. Tal composición de la dieta no fue asociada al tipo de tratamiento. Conclusión: En el grupo estudiado, el tipo de tratamiento antineoplásico no tuvo influencia en la situación nutricional y alimentaria detectada.

Palabras clave: Neoplasias de la Mama/radioterapia; Neoplasias de la Mama/quimioterapia; Consumo de Alimentos; Estado Nutricional; Dieta; Antropometría 